\title{
Tracheal injury in a toddler
}

E Motloung, ${ }^{1} \mathrm{MB}$ ChB, MMed, FCPS (SA), Cert Peads Nutrition (Boston); S Mapholi, ${ }^{1}$ MB ChB;

A B van $\mathbf{A s},{ }^{2} \mathrm{MB} C h B, \mathrm{FCS}(\mathrm{SA}), \mathrm{MMed}, \mathrm{MBA}, \mathrm{PhD}$

${ }^{1}$ Department of Paediatric Surgery, Mankweng Hospital, University of Limpopo, Polokwane, South Africa

${ }^{2}$ Department of General Surgery, Pietersburg Hospital, University of Limpopo, Polokwane, South Africa

Corresponding author: A B van As (sebastian.vanas@uct.ac.za)

Penetrating neck injuries are uncommon in the paediatric population. The initial management of these patients can be challenging to the emergency room physician, and the surgeon that performs the operative treatment. The nature of these injuries tends to be very complicated since, typically, the patient presents with either excessive bleeding or a compromised airway due to the damage of vital structures in the neck area such as the trachea and carotid-sheath, and lack of corresponding protection from the vertebral bone. Here, we report a traumatic case of a 2 -year-old girl that was referred to our unit after surviving an attempted murder by a deranged family member that slit her throat with a saw-toothed knife. The patient sustained a near complete slit of her throat, which was repaired primarily with satisfactory postoperative results.

S Afr Med J 2021;111(11b):1142-1144. https://doi.org/10.7196/SAMJ.2021.v111i11b.16100

A stab wound to the neck is a relatively uncommon condition in paediatrics. It is potentially dangerous due to the presence of vital structures in the neck and requires emergency management. The neck is made up of vital structures such as jugular veins and carotid arteries, the larynx and trachea, the oesophagus and the thyroid gland, all these structures are densely related. The management of penetrating neck injuries is clinically challenging as more than one of these structures are likely to be involved in a single incident. The mechanism of injury usually determines the extent of tissue damage and the treatment options. Stab wounds are relatively low-velocity injuries but can still lead to a life-threatening clinical scenario, requiring immediate surgical intervention.

\section{Case}

A 2-year-old girl was referred to the paediatric surgery unit at Polokwane/Mankweng Hospital from FH Odendal Hospital in Limpopo Province, South Africa. The history provided by a neighbour was that the child was stabbed on the neck by her mother, known to have severe mental health issues, who attempted to kill her children after a domestic dispute with their father.

On immediate inspection, the child arrived in respiratory distress, non-intubated and gasping for air. There was minimal bleeding in the area of the injury, and the child kept the neck in flexion to maintain her airway patent for breathing. On further assessment, the child was conscious but could not talk, and breathing with difficulty, with an oxygen saturation of $98 \%$ on room air.

The child was haemodynamically stable and pale with a pulse rate of 102 beats per minute, which was regular, and good volume.

The neck examination revealed a visible slit and open throat with saliva and air leaking through the wound (Fig. 1). The laceration was in the left and right anterior neck triangle with bilateral left and right sternocleidomastoid and thyroid-hyoid muscles completely cut. The trachea was visibly cut open anteriorly about $60-70 \%$, both the carotid arteries and jugular veins were bruised but not obviously perforated or bleeding. There was associated swelling at point of entry and tenderness with mild subcutaneous emphysema. Of note was a ragged appearance of skin and subcutaneous tissue without any major neurological deficit.
A blood gas, full blood count, electrolyte, urea, and creatinine analysis were performed, and all were normal. The patient was assisted by a specialist paediatric surgeon and anaesthesist, intubated while in the emergency unit and immediately taken to theatre for emergency surgery.

In theatre, the patient was placed in a supine position with the neck hyperextended. After draping, a neck exploration was performed, and the findings of the surgery were a completely severed right and left anterior neck triangle sternocleidomastoid muscle with $60-70 \%$ of the trachea circumference severed anteriorly (Fig. 2). The carotid arteries and jugular veins were both bruised, but not actively bleeding. The nerves and other organs of the anterior triangles such as the thyroid and thymus were all spared. The operative area was mechanically cleaned and irrigated repeatedly with saline, following which the trachea was sutured and repaired with 7 interrupted sutures using Vicryl 3.0 on a round needle (Fig. 3). Both the left and right anterior neck triangle muscles were approximated with Vicryl 3.0 and finally the skin was closed with Monocryl 4.0 (Fig. 4). The patient was taken to the paediatric intensive care unit (ICU) and intubated for both ventilation and stenting the trachea for 5 days.

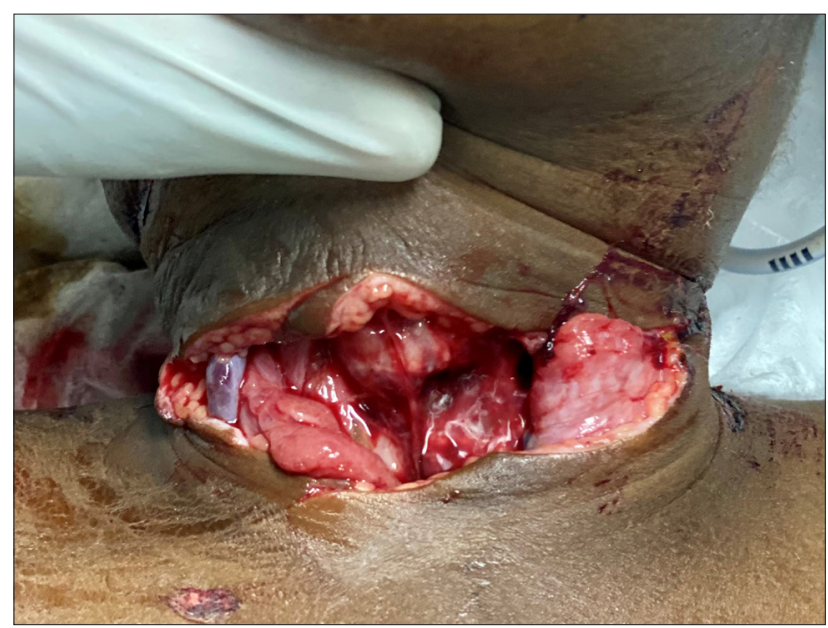

Fig. 1. Initial aspect of the anterior neck area on presentation. 


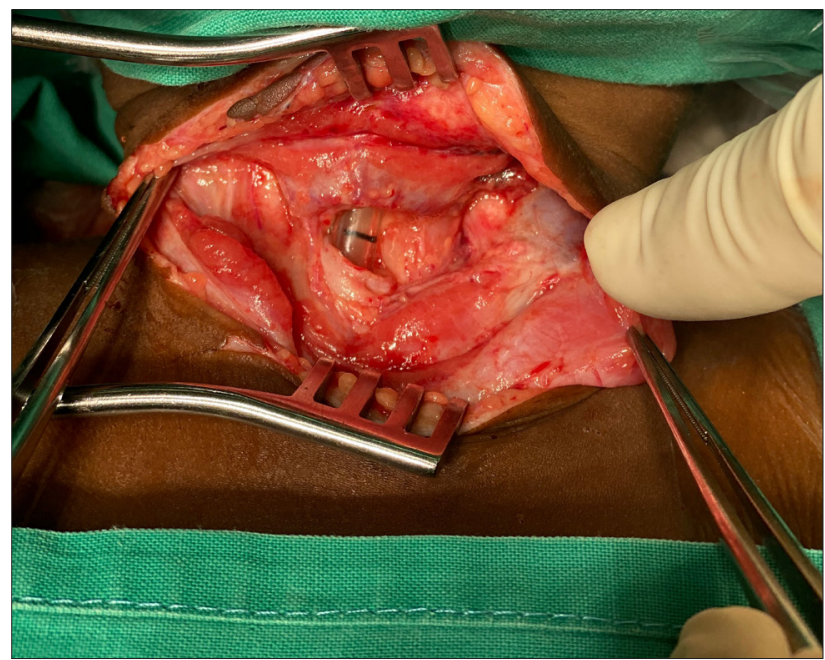

Fig. 2. Intraoperative view with the endotracheal tube visible in the severed trachea.

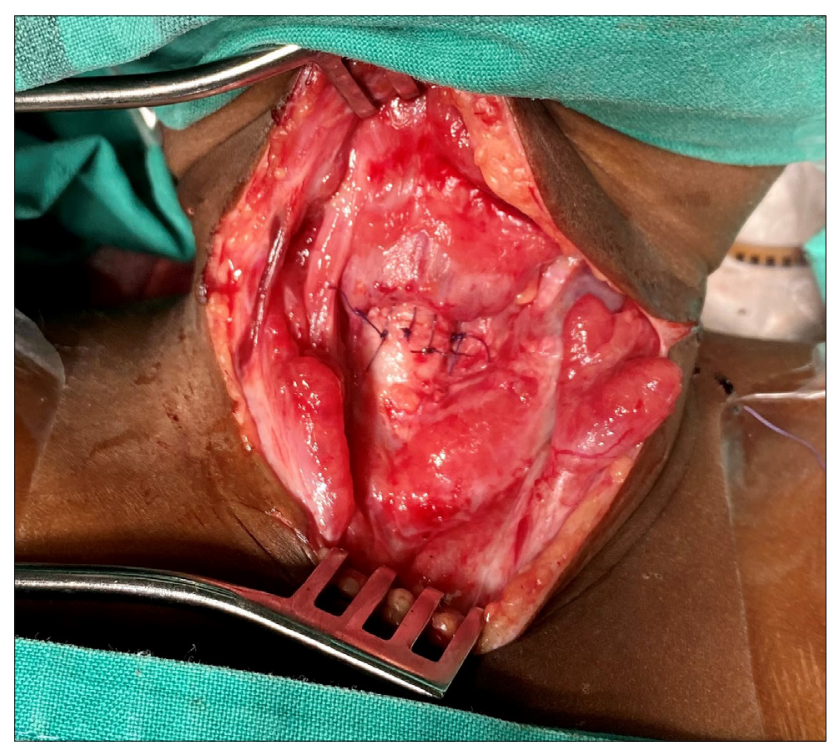

Fig. 3. View of the trachea after repair with interrupted vicryl sutures.

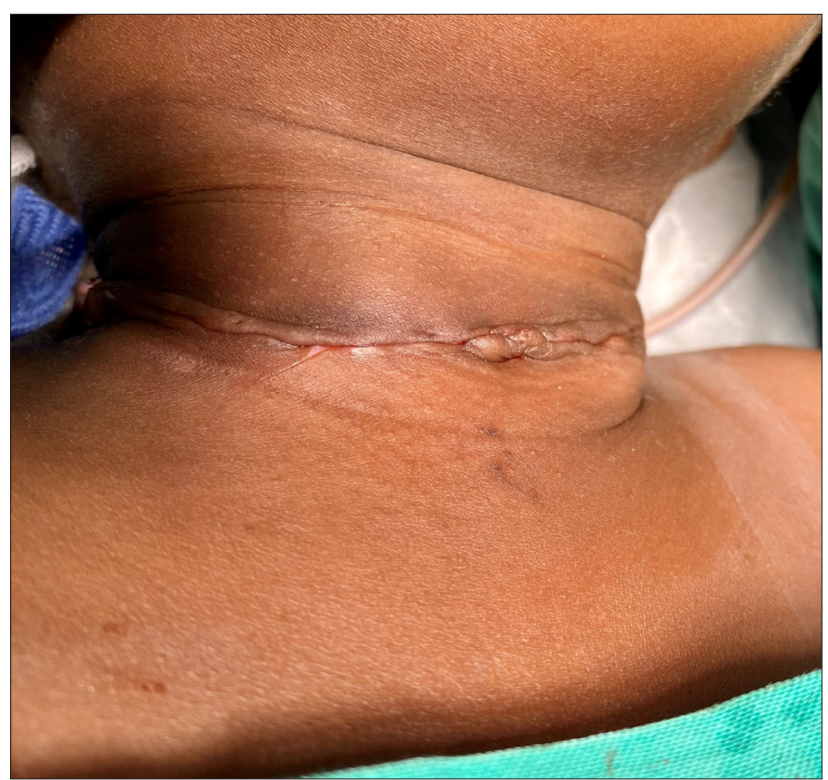

Fig. 4. Aspect of the anterior neck after closure of the skin.

\section{Discussion}

Penetrating neck injuries are defined as any injury that bridges the platysma muscle. They are potentially life-threatening injuries because of the vital structures in this critical area. In the paediatric population, they are uncommon injuries but when encountered, they prove to be challenging to the frontline medical personnel.

Children have a short neck hosting several important organs such as respiratory (larynx, pharynx), gastrointestinal (oesophagus), endocrine (thymus and thyroid gland), neuromuscular (nerves, carotid arteries, and jugular veins) and skeletal muscles. It is therefore a very difficult area with limited access for operative repair of damaged organs.

Penetrating injuries of the neck are considered life-threatening injuries as they can cause airway obstruction and severe bleeding resulting to hypovolaemic shock. Stab wounds to the neck are usually caused by sharp objects intentionally or unintentionally.

The most frequent symptoms are bleeding from the wound or the mouth due to injuries of the blood vessels, pharynx, larynx or oesophagus, or respiratory distress from tracheal injures depending on the zones of the neck penetrated.

In these types of injuries, it is paramount to secure the airway by intubation or tracheostomy to stop the bleeding by digital compression or catheter tamponade of the bleeding channel and early treatment of shock.

Most of these cases might require surgical exploration in the operating theatre, but in stable patients, imaging can be performed to evaluate the extent of the injuries.

The neck can be divided into three anatomical zones, and this can assist in the prognostication and management of penetrating neck wounds. Zone I extends from the lower border of the cricoid cartilage to the clavicles and thoracic outlet. Within this zone, the trachea, the great vessels, the oesophagus, the upper mediastinum, the lung apices, and the thoracic duct are located. Injuries in this zone have the highest mortality compared with the two other zones. Zone II includes the area between the cricoid cartilage and the angle of the mandible. Enclosed within this region are the carotid and vertebral arteries, jugular veins, pharynx, larynx, oesophagus, and trachea. Zone III involves the area above the angle of the mandible up to the base of the skull and includes the distal extracranial carotid and vertebral arteries as well as segments of the jugular veins. Injury to the index patient was between zone I and II of the neck, as the patient only suffered injuries to the sternocleidomastoid muscle and to the trachea while the carotid arteries and jugular veins were spared.

Prompt initial assessment and institution of management should be carried out according to the advanced trauma life support (ATLS) principles, prioritising the life-threatening conditions first. The ATLS guidelines established by the American College of Surgeons prioritise the airway, breathing and circulation control.

Management of penetrating neck injury ranges from surgical exploration based on the patient's clinical stability or radiological and endoscopy findings.

For stable patients, there is controversy in management algorithms, but the current trend is selective exploration as was followed in this case report. It should be noted that clinical indications for mandatory exploration include active bleeding, pulse deficit, increasing subcutaneous emphysema, haematemesis, haemoptysis, stridor, and worsening hoarseness.

Our patient was kept intubated in the paediatric ICU for 5 days, after which she was discharged to the general ward. The follow-up of the patient was uneventful as she developed no postoperative sequelae or tracheal stenosis. She was referred for post-traumatic counselling. 


\section{Conclusion}

This case report demonstrates that rapid resuscitation and early surgical management can be lifesaving and result in good clinical outcome.

Declaration. None.

Acknowledgements. None.

Author contributions. Equal contributions.
Funding. None.

Conflicts of interest. None.

1. Suleiman AO, Afolabi OA, Alabi BS. An unusual penetrating stab injury in a child: Case report. Int Pediatr Otorhinolaryngol Extra 2003;8(4):119-121. https://doi.org/10.1016/.jpedex.2013.07.004

2. Agrawal R, Timilsina DS, Reddy NS, Ganguly SN, Tayal A. Penetrating foreign body neck: Case report. Kathmandu University Med J 2007;5(3):414-416.

3. Moeng S, Boffar K. Penetrating neck injuries. Scand J Surg 2002;91:34-40. https:// doi:10.1177/145749690209100106

Accepted 3 September 2021 